\title{
Physical activity after total joint arthroplasty: a narrative review
}

This article was published in the following Dove Press journal: Open Access Journal of Sports Medicine

\section{Gustavo J Almeida Samannaaz S Khoja Sara R Piva}

Department of Physical Therapy, School of Health and Rehabilitation Science, University of Pittsburgh, Pittsburgh, PA, USA
Correspondence: Gustavo J Almeida Department of Physical Therapy, School of Health and Rehabilitation Science, University of Pittsburgh, Bridgeside Point I, 100 Technology Drive, Suite 210, Pittsburgh, PA I5219, USA

Tel +14126482215

Fax + I 4126485970

Email gja4@pitt.edu
Background: Total joint arthroplasty (TJA) is a common procedure to treat individuals with hip and knee osteoarthritis. While TJAs are successful in decreasing pain and improving quality of life, it is unclear whether individuals who undergo TJA become more physically active after surgery. It is possible that TJA, by itself, is not sufficient to affect the behavior of patients toward physical activity (PA) participation. To increase PA participation, individuals with TJA may need to be exposed to exercise/behavioral interventions specifically aimed to promote PA (ie, in addition to the surgery).

Objectives: This narrative review aimed to assess the evidence on 1) whether TJAs change PA participation from pre- to postsurgery and 2 ) whether exercise/behavioral interventions delivered before or after TJA help to promote PA in these patients.

Results: For aim 1, the studies that assessed PA from pre- to post-TJA reported that PA does not change in the first 3 months postsurgery. The results of follow-ups longer than 3 months but shorter than 12 months are contradictory, and the results of follow-ups longer than 12 months provide weak evidence of increased PA. Assessment of changes in PA due to TJA is challenged by the wide variability in demographics, methods used to assess PA, and different pathways of care used across studies. The results for aim 2 were limited by a scarcity of studies that used exercise/behavioral interventions to promote PA.

Conclusion: TJA relieves joint pain and offers a unique opportunity for patients to become more physically active. However, the current evidence is limited and unable to offer definitive results of whether TJA is effective to change PA from pre- to postsurgery. Future large studies in representative samples of patients with TJA are needed to adequately answer this question. Keywords: osteoarthritis, total knee arthroplasty, total hip arthroplasty, health promotion, quality of life, physical function, behavioral intervention

\section{Introduction}

Total joint arthroplasties (TJAs) of the hip and knee are among the most common elective surgical procedures in the USA. ${ }^{1}$ Due to the rapid growth in volume, TJAs pose a large economic burden in global healthcare systems. In the USA, these surgeries constitute the largest hospital expenditure category for Medicare. ${ }^{2}$ Despite the economic burden, TJAs have been clearly shown to successfully decrease pain and improve mobility and quality of life. However, it is unclear whether individuals who undergo TJAs become more physically active after surgery.

Individuals who undergo TJA tend to be older sedentary adults, and it is estimated that around $49 \%$ of them are overweight or obese, $16 \%$ have diabetes, and about half have high blood pressure. ${ }^{3,4}$ As TJA relieves joint pain, the pain-free state following 
surgery represents an opportunity for those individuals to become more physically active. Regular physical activity (PA) is one of the most effective interventions to improve the prevalent chronic comorbid conditions, such as obesity, diabetes, and hypertension, that commonly coexist with hip and knee osteoarthritis. ${ }^{5}$ Therefore, in addition to symptom relief, if TJA also increased PA, it could potentially benefit the overall health of the individual undergoing the surgery. ${ }^{6}$ However, it is unclear whether TJA increases PA from preto postsurgery, which we tried to clarify by reviewing the literature on this topic.

It is well known that changing human behavior, such as increasing PA, is very challenging. There is a possibility that TJA by itself may not be sufficient to change the patients' behavior toward PA participation. To increase PA participation, individuals with TJA may need to be exposed to interventions specifically aimed to promote PA (ie, in addition to surgery). Although there is a large body of literature about exercise or behavioral interventions in TJA that are administered to improve the outcome of these surgeries, it is unclear what interventions have been used and whether they are effective to promote PA. In this context, this review also proposes to appraise the literature on the use of exercise and behavioral interventions to promote PA in individuals post-TJA.

The first aim of the current review was to assess the evidence on whether TJAs change PA participation from pre- to postsurgery, and the second aim was to assess whether exercise/behavioral interventions delivered before or after TJA help to promote PA. This review also discusses the challenges related to promoting $\mathrm{PA}$ in this population.

\section{Methods}

We searched several databases including PubMed, EMBASE, Cochrane, CINAHL, and SPORTDiscus from inception until September 2017. Studies were included if they had a longitudinal design and assessed PA before and after total hip arthroplasty (THA) and total knee arthroplasty (TKA). Studies that assessed PA before and after exercise programs or promoted PA in patients with THA and TKA were also included. To search for relevant studies to address the first aim, the following terms were combined: ("arthroplasty, replacement, knee" [MESH] OR “arthroplasty, replacement, hip" [MESH]) AND ("exercise" [MESH] OR "physical activity"). Then, the same terms were combined with "exercise OR behavioral OR intervention" for the search related to the second aim. Two authors (GJA and SSK) reviewed all titles and abstracts identified through the search strategy and retrieved the full text when a study seemed appropriate to be included in this review. The two authors also hand searched the reference lists of articles to identify potential studies not detected by the search strategy. A total of 714 titles and abstracts were reviewed. The majority of the studies found in the literature search have assessed outcomes of pain, range of motion, physical function or quality of life, but not PA. Most studies that assessed outcome measures from preto post-TJA were excluded from this review, as they did not assess PA. Similarly, most studies that assessed outcomes from pre- to postexercise/behavioral intervention were excluded for not assessing PA. From our search strategy, we found 17 articles that met our inclusion criteria and were included in this narrative review: 14 articles reported on PA participation pre-/post-TJA ( six in THA only, five in TKA only, and three in THA and TKA combined), and three articles reported on PA before and after exercise/behavioral intervention in individuals with TJA (all in TKA).

\section{Results}

The studies that met inclusion criteria were highly variable in terms of length of follow-up and methods used to assess PA. The length of follow-up ranged from 6 weeks to 24 months postsurgery. The methods to assess PA also varied across studies, with some studies using either self-report questionnaires or real-time activity monitors, or the combination of both. The studies that used self-report questionnaires queried about a wide range of activities and reported PA scores in a total PA score, energy expenditure per week, or time spent in each activity per week. Among studies that used real-time activity monitors, some measured daily step counts, while others measured energy expenditure during PA or time spent in different PA intensities. Therefore, to compare changes in PA across studies, study findings were organized based on the method used to measure PA. We organized the results by self-report PA questionnaires and real-time activity monitors as self-report questionnaires are known to overestimate PA as compared to real-time activity monitors. ${ }^{7,8}$ We also organized the results based on surgical joint (THA and TKA) because functional outcomes are known to differ between THA and TKA. ${ }^{9,10}$ Then, to effectively compare changes in PA across studies, we created a common metric. We calculated the percentage improvement in PA: difference between follow-up (postsurgery) and baseline (presurgery) PA levels divided by baseline PA.

\section{Studies that assessed PA pre- and post- THA}

Nine studies examined PA participation pre-/post-THA (Table 1), in which four measured PA using an activity monitor, ${ }^{11-14}$ two used a self-report questionnaire, ${ }^{15,16}$ and 


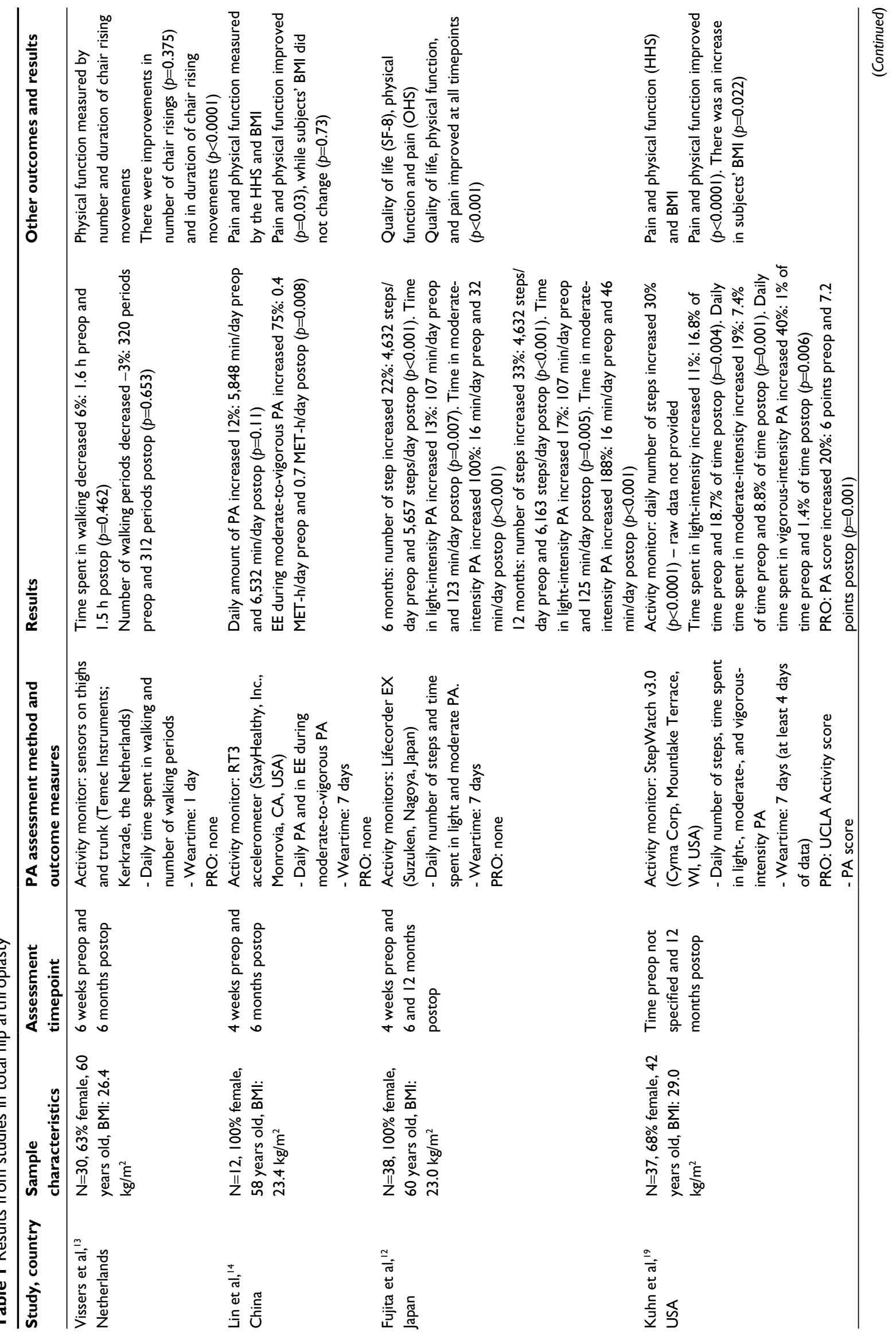











Open Access Journal of Sports Medicine 20I8:9 
Three studies used both activity monitor and self-report questionnaire. de Groot et $\mathrm{al}^{17}$ assessed changes in PA at 3 and 6 months postsurgery in 36 subjects (average age 62 years) who underwent THA. Subjects wore an activity monitor with three sensors (one attached to the trunk and two to both thighs). The study reported no changes in time spent in movement-related activity $(5 \%-6 \%, p \geq 0.22)$ as assessed by the activity monitor at 3 or 6 months.

Results from self-reported PA indicated a nonsignificant increase of $20 \%$ ( 1.7 points in PA score, $p=0.19)$ at 3 months follow-up and a significant increase of $98 \%$ (7.6 points in PA score, $p<0.001$ ) at 6 months. ${ }^{17}$ Harding et al ${ }^{18}$ investigated changes in PA at 6 months post-THA in 19 patients (average age 69 years). Subjects wore an activity monitor on their waist. There was a nonsignificant decrease of $13 \%$ in PA ( 16 counts per minute, $p=0.06$ ) as measured by the activity monitor. However, there was a significant increase of $33 \%$ in self-reported PA ( 1 point in PA score, $p<0.001) .{ }^{18} \mathrm{Kuhn}$ et $\mathrm{al}^{19}$ assessed changes in PA at 12 months post-THA in 37 patients (average age 42 years). Subjects wore an activity monitor attached to the lateral side of the ankle. The study results demonstrated a significant increase of 30\% in step count (1,043 steps/day, $p<0.0001), 11 \%$ in time spent in lightintensity PA (1.9\%/day, $p=0.004), 19 \%$ in moderate-intensity PA $(1.4 \%$ day, $p=0.001)$, and $40 \%$ in vigorous-intensity PA $(0.4 \% /$ day, $p=0.006)$. Self-reported PA also increased by $20 \%$ (1.2 points in PA score, $p=0.001){ }^{19}$

\section{Studies that assessed PA pre- and post- TKA}

Eight studies investigated PA participation pre-/post-TKA (Table 2). Three studies used an activity monitor, ${ }^{27-29}$ one used a self-report questionnaire, ${ }^{16}$ and four used both. ${ }^{17,18,30,31}$ Schotanus et $\mathrm{al}^{27}$ investigated changes in PA at 6 weeks postsurgery in 20 subjects (average age 66 years) who underwent TKA. PA was measured using an activity monitor attached to the lateral side of the nonsurgical upper leg. The study reported a significant decrease in number of steps by $35 \%$ $(-2,600$ steps/day, $p<0.05)$ and nonsignificant decreases in the percentage of time spent in PA by $24 \%(-11 \%$ of daily activities, $p \geq 0.05) .{ }^{27}$ Brandes et $\mathrm{al}^{28}$ examined changes in PA at 2, 6, and 12 months postsurgery in 53 subjects (average age 66 years) who underwent TKA. PA was measured using two activity monitors: one that combines sensors worn around the waist and trunk and another mounted to the subjects' right ankle. Number of steps decreased by $6 \%$ at 2 months follow-up ( -526 steps/day, $p \geq 0.05$ ) and increased significantly by $10 \%(1,006$ steps/day, $p=0.03)$ and $19 \%$
(1,878 steps/day, $p=0.003$ ) at 6 and 12 months follow-ups, respectively. Results also indicated no significant changes at 2 months $(17 \% ; 1.4 \% /$ day, $p \geq 0.05)$ in percentage of time spent in locomotion, but significant increases of $43 \%$ were found for both 6 and 12 months follow-ups (3.6\%/day, $p<0.01)$. Percentage of time in sedentary activities (ie, resting) did not change significantly at 2 months $(7 \% ; 4.7 \%$ day, $p \geq 0.05)$ but decreased at 6 and 12 months by $15 \%(9.7 \% /$ day, $p<0.05)$ and $16 \%$ (10.5\%/day, $p<0.01)$, respectively. ${ }^{28}$ Lutzner et $\mathrm{al}^{29}$ investigated changes in PA at 12 months postsurgery in 221 subjects (average age 68 years) who underwent TKA. PA was measured using an activity monitor on the lateral side of the lower leg. The study reported a significant increase of $23 \%(1,216 \mathrm{steps} /$ day, $p=0.001)$ in total number of steps and a $60 \%$ increase (686 steps/day, $p=0.001$ ) in number of steps at moderate intensity. However, daily time in sedentary activities (ie, lying, sitting, and standing) did not change at 12 months follow-up $(2 \%, p \geq 0.203){ }^{29}$

One study assessed changes in PA using a self-reported questionnaire. Smith et $\mathrm{al}^{16}$ analyzed data from the Osteoarthritis Initiative data set to investigate changes in PA at 12 and 24 months postsurgery in 116 subjects (average age 67 years) who underwent TKA. Results showed no change in PA at 12 months (change of $0.7 \%$ in PA score, $p=0.93$ ); however, there was an increase in PA of $13 \%$ (16 points in PASE score, $p=0.04$ ) at 24 months postsurgery. ${ }^{16}$

The four studies that assessed PA using both activity monitor and self-report questionnaire reported diverging results between measurement methods. Walker et a ${ }^{30}$ investigated changes in PA at 3 and 6 months postsurgery in 19 subjects (average age 69 years) who underwent TKA. Subjects wore an activity monitor with two sensors (one attached to the thorax and another to the lateral side of the lower leg) and results from the activity monitor showed a nonsignificant increase in ambulatory energy expenditure (calculated as amplitude multiplied by number of steps) at 3 months post-TKA $(29 \% ; 65,000, p \geq 0.05)$. However, the increase was $79 \%$ and significant at 6 months post-TKA $(175,000$, $p=0.01)$. The same was observed for number of steps where they reported a nonsignificant increase of $15 \%$ (1,620 steps/ day, $p \geq 0.05$ ) at 3 months and a significant increase of $46 \%$ (4,900 steps/day, $p=0.01)$ at 6 months post-TKA. Results from self-reported PA indicated a significant increase in energy expenditure at 3 months ( 31 points, $63 \%, p<0.05$ ) and 6 months ( 30 points, $61 \%, p<0.05$ ). ${ }^{30}$ Tsonga et $\mathrm{al}^{31}$ examined changes in PA at 3 and 6 months postsurgery in 52 subjects (average age 73 years) who underwent TKA. Subjects wore the activity monitor around the waist, and the results showed 


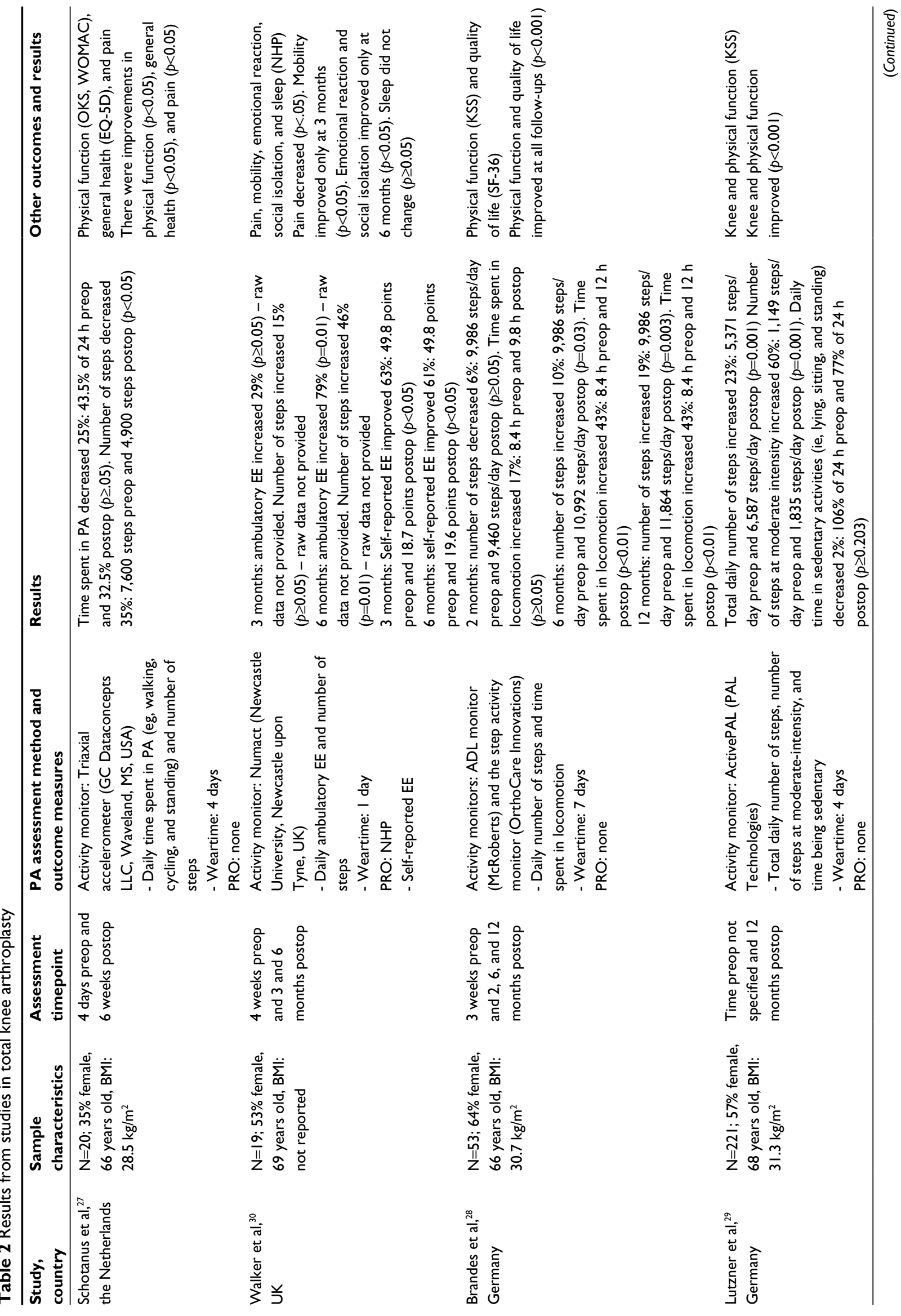




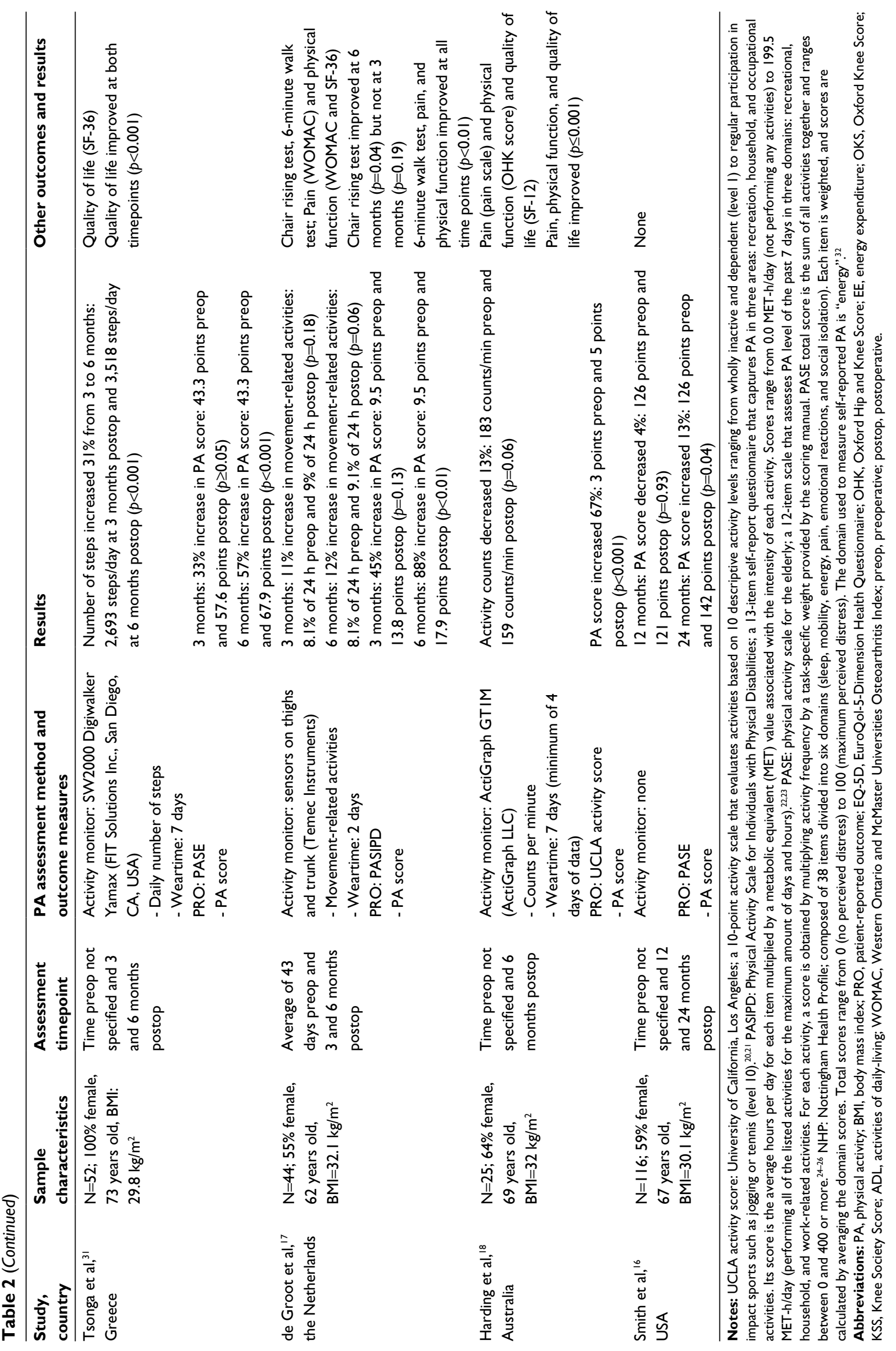


significant increase of $31 \%$ in number of steps from 3 to 6 months post-TKA (825 steps/day, $p<0.001$ ). Results from self-reported PA indicated a nonsignificant increase of $33 \%$ at 3 months ( 14.3 points in PA score, $p \geq 0.05$ ), but significant increase of $57 \%$ at 6 months post-TKA (24.6 points in PA score, $p<0.001) .{ }^{31}$ de Groot et $\mathrm{al}^{17}$ investigated changes in PA at 3 and 6 months postsurgery in 44 subjects (average age 62 years) who underwent TKA. The activity monitor used consists of three sensors (one attached to the thorax and two to the lateral side of both thighs), and it reported nonsignificant increases in movement-related activities at 3 months $(11 \%$, $p=0.18)$ and 6 months $(12 \%, p=0.06)$ post-TKA. Results from self-reported PA showed no significant increase of $45 \%$ at 3 months post-TKA (4.3 points in PA score, $p=0.13$ ) and a significant increase of $88 \%$ at 6 months ( 8.4 points in PA score, $p<0.01) .{ }^{17}$ Harding et $\mathrm{al}^{18}$ investigated changes in PA at 6 months postsurgery in 25 subjects (average age 69 years) who underwent TKA. Subjects wore the activity monitor around the waist, and they reported a nonsignificant decrease of $13 \%$ ( 24 counts $/ \mathrm{min}, p=0.06$ ). This study also measured time spent in sedentary behavior and reported that subjects spent $80 \%$ of their day being sedentary, which remained the same at 6 months postsurgery. Self-reported PA increased significantly by $67 \%$ ( 2 point in PA score, $p<0.001)$ at 6 months follow-up. ${ }^{18}$

\section{Studies that assessed PA pre- and postexercise or had a behavioral intervention in TJA}

The literature search did not identify studies that addressed PA promotion using an exercise program or behavioral approaches in THA. We found three studies in TKA that measured PA as an outcome and included exercise or PA promotion as part of the intervention ${ }^{33-35}$ (Table 3). Moffet et $\mathrm{al}^{33}$ compared the effect of a supervised rehabilitation program to standard of care in 77 subjects (average age 68 years) who were between 2 and 4 months post-TKA. The rehabilitation program consisted of 12 supervised sessions carried out over 6-8 weeks and included strengthening exercises of the lower extremity, endurance exercises (cycling or walking), and exercises that mimicked functional tasks such as sit-tostand and stair climb. While no aspects of the rehabilitation program specifically focused on promoting PA during daily routine, the investigators queried the participants on their PA levels during baseline, on completion of the supervised rehabilitation program and at the 6 months follow-up. The study reported no differences between the intervention and the standard of care groups on level of PA at 4 and 6 months.
The magnitude of changes in PA from baseline to 6 months post-TKA was not reported. ${ }^{33}$

Monticone et $\mathrm{al}^{34}$ compared the effects of a home exercise program combined with advice to stay active versus advice to stay active only in 110 subjects (average age 68 years) who had undergone TKA. Individuals were enrolled into the study upon discharge from a 15-day inpatient rehabilitation program postsurgery. This study included an educational component geared toward promoting PA; however, there were no PA measures collected pre- or poststudy intervention. Hence, this limited the ability to conclude whether the study intervention was successful in increasing PA post-TKA. ${ }^{34}$

Piva et $\mathrm{a}^{35}$ compared a comprehensive intervention that combined high-intensity exercises with a PA promotion component to a standard of care exercise program in 44 subjects (average age 68 years) who were at least 2 months post-TKA. The PA promotion component mainly consisted of education and goal setting to encourage PA participation in patients' daily routine. This was the only study on rehabilitation post-TKA that included an objective assessment of PA using accelerometry. The authors reported a small improvement of $7 \%$ ( 9 minutes of daily PA) at 6 months follow-up in the group that received the PA promotion, while those in the standard exercise program decreased their daily PA by 14\% (30 minutes a day). The difference between the groups was not significant. ${ }^{35}$

\section{Discussion}

The first objective of this review was to assess whether there are changes in PA behavior due to TJA. The combined results from the studies included in this review are inconclusive. One consistent observation across studies in THA and TKA was that PA does not change during the first 3 months after surgery. In addition, there is a weak evidence that PA increases 12 months or longer after TKA, but this finding is not consistent in THA. ${ }^{16,29}$

The discrepancy in findings across studies may be dependent on several factors. This review indicates that time of follow-up after surgery may be one such factor, and it is likely that PA would increase as months go by after surgery. For example, the only study that assessed PA at 6 weeks postsurgery (TKA) reported significant decreases in PA (Schotanus et al), ${ }^{27}$ which could be explained by the presence of postsurgical pain and discomfort. The studies that measured PA from 6 weeks to 3 months postsurgery consistently reported findings of no changes in PA across THA and TKA populations. ${ }^{11,15,17,27,28,30,31}$ At 6 months postTJA, findings were variable: five studies reported increases 


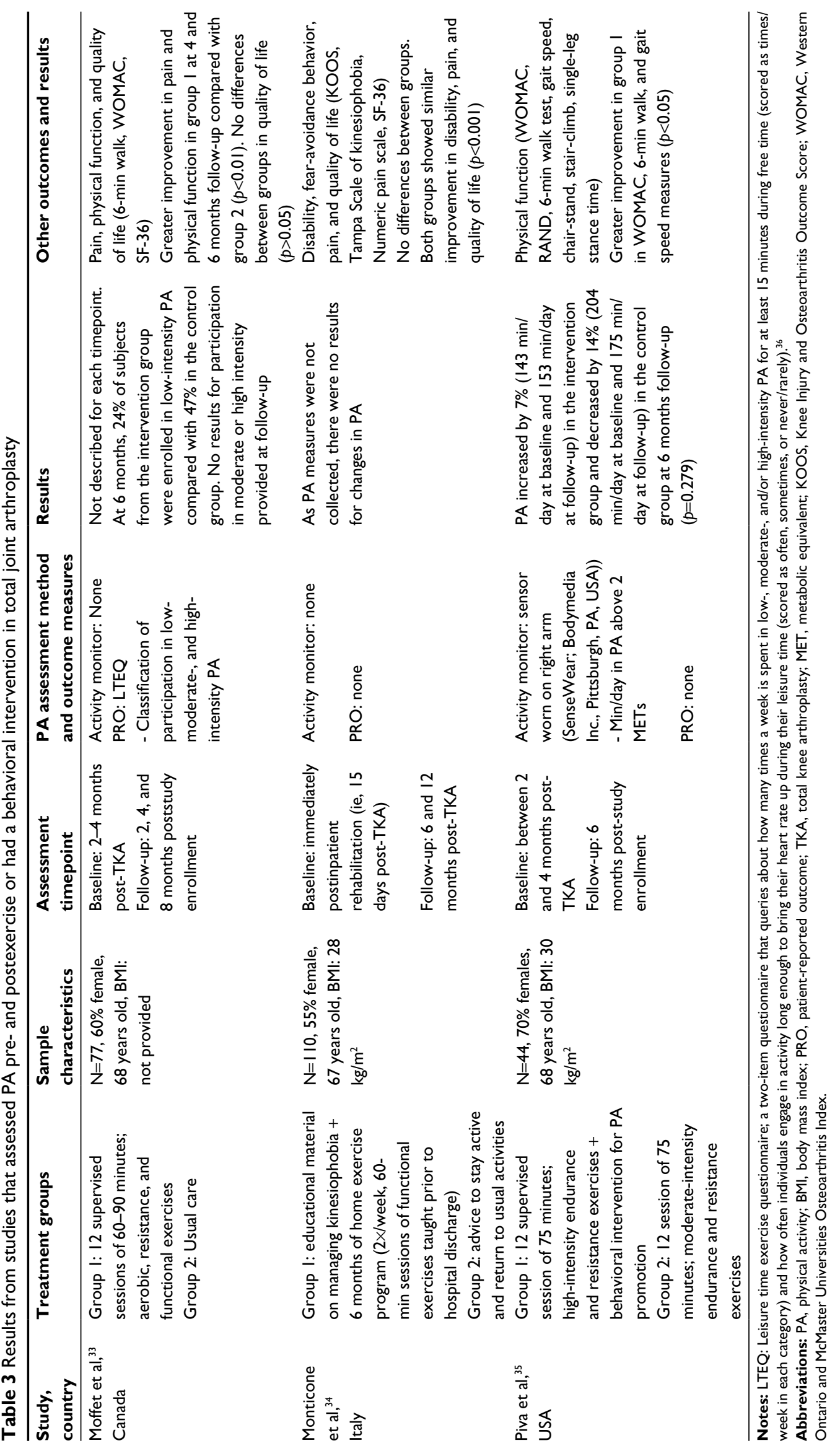


in PA, ${ }^{12,15,28,30,31}$ one reported no change, ${ }^{13}$ and three others reported increases in one PA outcome but not in the other (eg, self-reported PA versus real-time PA). ${ }^{14,17,18}$ Findings from studies with follow-ups of 12 months or longer were also variable, with a trend toward increased PA (six reporting increases ${ }^{12,15,16,19,28,29}$ and two reporting no changes in $\left.\mathrm{PA}^{11,16}\right)$. It was interesting to note that none of the studies with longer follow-ups reported decreases in PA. Reports on increases in PA ranged from $10 \%$ to $100 \%$ at 6 months after surgery $12,14,15,17,18,28,30,31$ and from $11 \%$ to $188 \%$ at 12 months. ${ }^{12,15,19,28,29}$ Thus, it may take several months for tissues to heal after surgery and for patients to feel comfortable to participate in PA. Although it is known that the functional recovery from TKA is slower than that from THA, we did not observe any differential trends in PA changes over time between the two cohorts of subjects.

Another factor for the discrepant findings is the different methods used to measure PA. The studies that used both activity monitors and self-reported methods to measure PA reported conflicting findings. For example, three studies assessed PA during the 6 months follow-up (one in TKA and two included both TKA and THA cohorts), and in general, they reported larger changes for self-reported compared to objectively measured PA..$^{14,17,18,23}$ These discrepancies were likely due to the well-known overestimation of self-reported PA as compared to real-time PA measures. ${ }^{78}$ In addition, most studies did not indicate whether the tools used to assess PA were reliable, valid, or responsive. In general, PA is highly variable and can change on a daily basis depending on an individual's routine and behavior. ${ }^{37}$ Therefore, reliable and valid tools are key for consistent and accurate assessment of PA, whereas responsive tools are needed to capture changes over time. Using measurement tools with poor psychometric properties may have contributed to the discrepant findings.

Diversity in the study design and the population studied may also help explain discrepant findings. For example, while most studies included consecutive patients, some did not provide details about recruitment and selection or adherence to wearing activity monitors, raising concerns about selection bias. Another concern was retention bias because one study reported a retention rate lower than $70 \% .{ }^{12}$ As several studies did not report on adherence to wearing monitors, it is likely that they excluded subjects in whom PA outcomes were insufficient at the follow-up assessment. Because of lack of information, it was not possible to evaluate whether retention rates or adherence to wearing activity monitors affected individual study findings. In addition, most (seven of nine) studies had sample sizes $<40$, which may have precluded the identification of significant changes in PA. In terms of patient population, it is important to note the diverse geographic (eg, Japan, China, Australia, and the USA) and gender distribution. For example, the study that reported the largest increases in PA (30 min/day of moderate intensity measured by an activity monitor) at 12 months was done in 38 Japanese women; ${ }^{12}$ this finding contrasts with a study in 105 Americans (men and women) that reported no change in self-reported PA at 12 months although self-reports generally overestimate PA participation. ${ }^{16}$ Because the prevalence of obesity in the USA is higher than that in Japan (around 34\% and $4 \%$, respectively) and men are more physically active than women, ${ }^{38}$ gender and nationality are also likely to contribute to the divergent findings. Changes in PA may also be impacted by the age of the individuals studied in the different cohorts. However, it did not seem that changes in PA from pre- to post-TJA were associated with age. When comparing the findings across studies that had 12 months follow-up postsurgery, the study with the youngest cohort (average age 42 years) reported $30 \%$ increase in number of steps, ${ }^{19}$ which was within the range presented by studies that included older subjects (60-68 years of age): $16 \%-33 \%$ improvement in number of steps. ${ }^{11,12,28,29}$ Although the study by Kuhn et al ${ }^{19}$ (younger sample) was in the upper range of changes, it was the only study in a younger population, which limits the ability to assess whether age impacted changes in PA.

The second objective of this review was to assess whether exercise or behavioral interventions (not the TJA surgery) may affect PA participation. We found that the evidence on effectiveness of exercise/behavioral interventions to improve PA post-TJA is extremely scarce and inconclusive, with none of the studies showing significant changes in PA after intervention. We were surprised that no studies investigated behavioral approaches to promote PA in THA and that only three studies did so in TKA. Of the three studies in TKA, only one (Piva et al) ${ }^{25}$ included a behavioral approach to improve PA and measured PA in real time. However, it did not observe significant differences between those exposed and not exposed to the PA promotion program, which may be due to the difficulty in changing individuals' behavior toward an active lifestyle.

The findings of this review are also supported by a large observational study that compared PA behavior in multiple cohorts of patients at different timepoints from joint surgery. Lubbeke et a ${ }^{39}$ conducted a cross-sectional analysis to compare PA in a cohort of patients who were about to undergo THA (these patients were asked to self-report their PA 2 weeks before surgery and also to recall their PA before the 
onset of osteoarthritis symptoms) with a cohort of patients who were 5 years post-THA and a cohort of patients who were 10 years post-THA. As this study did not assess longitudinal changes in PA pre- and post-THA within the same cohort of patients, it was not included in our review. However, the findings of Lubbeke et al are seminal because they provide a snapshot of how PA levels compare in a representative sample of patients at different timepoints across the spectrum of the osteoarthritis condition from prior to onset of symptoms to 10 years post-THA (189 patients prior to onset of symptoms, 203 patients prior to THA, 1,085 patients at 5 years after surgery, and 757 patients at 10 years after surgery). ${ }^{39}$ Results from Lubbeke et al $^{39}$ also illustrate that the surgery may be limited in its ability to encourage an active lifestyle. PA participation was highest prior to onset of osteoarthritis symptoms and lowest 2 weeks prior to THA. PA levels increased slightly at 5 and 10 years post-THA but did not reach the levels seen in those prior to onset of osteoarthritis symptoms. ${ }^{39}$ Larger observational longitudinal studies with better design are needed to provide more definitive answers of whether PA changes after TJA. Studies should have broad inclusion/ exclusion criteria, geographic variation, PA measurements with adequate psychometrics, and sufficient follow-up (ie, at least 2 years after surgery).

Although our review was comprehensive, we acknowledge that it was a narrative review and not a systematic review. Therefore, the quality of the included studies and the heterogeneity of study results were not assessed with the standardized rigor upheld in systematic reviews. In addition, we did not differentiate studies that assessed PA using 1 day from those using $\geq 5$ days, which could have had implications for the reliability of PA measures. However, in comparing the results across studies, we could not find a trend indicating that data collected using fewer days had less or more changes in PA. Another limitation may be the use of percentage change to compare results across studies. Although it is a method acceptable to compare study findings, this method is highly affected by low baseline values; even small changes in individuals with low baseline values of PA will represent very large percentage changes.

\section{Considerations for promoting PA in individuals with TJA}

TJA presents a unique opportunity for patients with pain due to end-stage joint disease to become more active as the surgery is successful in relieving pain and improving physical function. Promoting PA in this population is important to manage comorbid conditions that are present in many of the individuals living with TJAs. However, symptoms relief and improved physical function do not seem sufficient to promote an active lifestyle. The results of a recently published meta-analysis corroborate our findings as it demonstrated significant improvements in physical function and quality of life between 6 and 12 months post-TJA, but not in PA. ${ }^{40}$ Results from that meta-analysis offer limited evidence that subjects after TJA tend to increase their PA participation 12 months or later after surgery. Therefore, to promote PA, it may be necessary to expose individuals with TJA to behavioral and exercise programs that specifically target PA.

To effectively promote PA, it is important to first understand the barriers to PA on individual and societal levels, which may not be limited to disability or joint symptoms. The literature has identified several barriers to PA, which can be broadly classified into personal barriers (eg, expectations, motivation, unwillingness to change) and environmental barriers (eg, no access to exercise facilities, no sidewalks in the neighborhood). Individuals usually decide to undergo TJA expecting to resolve pain and functional limitations experienced for years due to osteoarthritis. However, many years of chronic pain may contribute to the development of negative behaviors such as pain catastrophizing and fear and avoidance of activity that may hamper adopting an active lifestyle. These behaviors may be compounded by barriers such as lack of social support and difficult access to exercise facilities and result in a sedentary lifestyle very resistant to change.

Barriers to PA in the TJA population can also manifest within the health care environment. Many individuals with TJA are cautioned by their physicians not to do too much activity (ie, vigorous intensity or high impact) as it may lead to excessive wear and tear, premature prosthesis failure, and consequent revision surgery. This cautious attitude toward PA may also generate fear of movement, which encourages already sedentary individuals to remain inactive. Rehabilitation clinicians may also be overly cautious and inadequately or under prescribe exercise programs during recovery from surgery. Under-dosed exercises may not sufficiently improve patients' physical function to the level necessary for them to be confident in participating in more biomechanically demanding activities. While activities that cause great stress on the hip or knee joints, such as jumping or running, should be discouraged, there is no evidence that high-level resistance training and low-impact aerobic activities such as swimming, brisk walking, and cycling decrease the life span of TJA. ${ }^{41}$ Furthermore, the trade-off between the harms of inactivity versus the benefits of PA, particularly considering the age and associated comorbidities of these patients, should be 
balanced. However, health care providers do not always have sufficient time to educate patients about the vast benefits of regular PA, ways to keep active, and types of activities that can be performed safely and are not deleterious to the prosthesis. A positive shift in attitude toward promoting PA, rather than a cautious attitude, along with provision of education about safe PA may be beneficial and the first step in encouraging an active lifestyle in this population.

Current clinical practice after TJA does not effectively promote PA participation. While there is emphasis on the physical approaches (ie, structured exercise program with a focus on reducing physical impairment or functional limitations), there is no sufficient attention on the behavioral aspect of PA participation. PA promotion requires behavioral interventions that aim to empower an individual to become more active by changing his/her lifestyle. PA promotion requires a team-based approach, which may include primary care physician, nurse, orthopedic surgeon, physical therapist, or exercise counselor. The behavioral approaches need to be applied in a systematic manner and involve measuring PA using instruments with good psychometric properties and include self-management strategies that set goals and review achievements. Research has also shown that patients need constant reminders to keep active. Prompts generated by a smartphone via text message, activity trackers, or regular phone calls have shown to be effective and could be incorporated in interventions to promote PA. ${ }^{42-44}$ The success of a PA promotion program also depends on the patient's willingness to change. One of the recommended components of a PA promotion is assessing the patient's readiness to change. ${ }^{45}$ Research has shown that applying a behavioral intervention to individuals who are unwilling to or uninterested in change may result in failure to change behavior despite the efforts put by the team involved in the process. ${ }^{46,47}$ Given all the positive effects of an active lifestyle and the complexity of modifying individual habits, studies need to identify patients who are undergoing TJAs that may benefit from PA promotion (ie, those motivated and willing to change behavior) and test the feasibility of the intervention, its acceptability, and effectiveness.

\section{Disclosure}

The authors report no conflicts of interest in this work.

\section{References}

1. Kurtz SM, Ong KL, Schmier J, Zhao K, Mowat F, Lau E. Primary and revision arthroplasty surgery caseloads in the United States from 1990 to 2004. J Arthroplasty. 2009;24(2):195-203.

2. Fischer ES, Bell JS, Tomek IM, Esty AR, Godman DC. Trends and Regional Variation in Hip, Knee, and Shoulder Replacement. Hanover: The Dartmouth Institute for Health Policy and Clinical Practice; 2010.
3. Murphy L, Helmick CG. The impact of osteoarthritis in the United States: a population-health perspective: a population-based review of the fourth most common cause of hospitalization in U.S. adults. Orthop Nurs. 2012;31(2):85-91.

4. Murphy L, Helmick CG. The impact of osteoarthritis in the United States: a population-health perspective. Am J Nurs. 2012;112(3 Suppl 1):S13-S19.

5. US Department of Health and Human Services (USDHHS). 2008 Physical Activity Guidelines for Americans. Washington, DC: USDHHS; 2008. http://www.health.gov/paguidelines/default/aspx. Accessed July $26,2017$.

6. Messier SP, Callahan LF, Golightly YM, Keefe FJ. OARSI Clinical Trials Recommendations: design and conduct of clinical trials of lifestyle diet and exercise interventions for osteoarthritis. Osteoarthritis Cartilage. 2015;23(5):787-797.

7. Neilson HK, Robson PJ, Friedenreich CM, Csizmadi I. Estimating activity energy expenditure: how valid are physical activity questionnaires? Am J Clin Nutr. 2008;87(2):279-291.

8. Almeida GJ, Irrgang JJ, Fitzgerald GK, Jakicic JM, Piva SR. Reliability of physical activity measures during free-living activities in people after total knee arthroplasty. Phys Ther. 2016;96(6):898-907.

9. Choi JK, Geller JA, Yoon RS, Wang W, Macaulay W. Comparison of total hip and knee arthroplasty cohorts and short-term outcomes from a single-center joint registry. J Arthroplasty. 2012;27(6):837-841.

10. Dailiana ZH, Papakostidou I, Varitimidis S, et al. Patient-reported quality of life after primary major joint arthroplasty: a prospective comparison of hip and knee arthroplasty. BMC Musculoskelet Disord. $2015 ; 16: 366$.

11. Jeldi AJ, Deakin AH, Allen DJ, Granat MH, Grant M, Stansfield BW. Total hip arthroplasty improves pain and function but not physical activity. J Arthroplasty. 2017;32(7):2191-2198.

12. Fujita K, Makimoto K, Tanaka R, Mawatari M, Hotokebuchi T. Prospective study of physical activity and quality of life in Japanese women undergoing total hip arthroplasty. J Orthop Sci. 2013;18(1):45-53.

13. Vissers MM, Bussmann JB, de Groot IB, Verhaar JA, Reijman M. Walking and chair rising performed in the daily life situation before and after total hip arthroplasty. Osteoarthritis Cartilage. 2011;19(9):1102-1107.

14. Lin BA, Thomas P, Spiezia F, Loppini M, Maffulli N. Changes in daily physical activity before and after total hip arthroplasty. A pilot study using accelerometry. Surgeon. 2013;11(2):87-91.

15. Rosenlund S, Broeng L, Holsgaard-Larsen A, Jensen C, Overgaard S. Patient-reported outcome after total hip arthroplasty: comparison between lateral and posterior approach. Acta Orthop. 2017;88(3):239-247.

16. Smith TO, Mansfield M, Dainty J, Hilton G, Mann CJV, Sackley CM. Does physical activity change following hip and knee replacement? Matched case-control study evaluating physical activity scale for the elderly data from the osteoarthritis initiative. Physiotherapy. 2017 Epub Sep 13.

17. de Groot IB, Bussmann HJ, Stam HJ, Verhaar JA. Small increase of actual physical activity 6 months after total hip or knee arthroplasty. Clin Orthop Relat Res. 2008;466(9):2201-2208.

18. Harding P, Holland AE, Delany C, Hinman RS. Do activity levels increase after total hip and knee arthroplasty? Clin Orthop Relat Res. 2014;472(5):1502-1511.

19. Kuhn M, Harris-Hayes M, Steger-May K, Pashos G, Clohisy JC. Total hip arthroplasty in patients 50 years or less: do we improve activity profiles? J Arthroplasty. 2013;28(5):872-876.

20. Zahiri CA, Schmalzried TP, Szuszczewicz ES, Amstutz HC. Assessing activity in joint replacement patients. J Arthroplasty. 1998;13(8):890-895.

21. Naal FD, Impellizzeri FM, Leunig M. Which is the best activity rating scale for patients undergoing total joint arthroplasty? Clin Orthop Relat Res. 2009;467(4):958-965.

22. Washburn RA, Zhu W, McAuley E, Frogley M, Figoni SF. The physical activity scale for individuals with physical disabilities: development and evaluation. Arch Phys Med Rehabil. 2002;83(2):193-200. 
23. van den Berg-Emons RJ, L'Ortye AA, Buffart LM, et al. Validation of the physical activity scale for individuals with physical disabilities. Arch Phys Med Rehabil. 2011;92(6):923-928.

24. Washburn RA, Smith KW, Jette AM, Janney CA. The Physical Activity Scale for the Elderly (PASE): development and evaluation. J Clin Epidemiol. 1993;46(2):153-162.

25. Svege I, Kolle E, Risberg MA. Reliability and validity of the Physical Activity Scale for the Elderly (PASE) in patients with hip osteoarthritis. BMC Musculoskelet Disord. 2012;13:26.

26. Bolszak S, Casartelli NC, Impellizzeri FM, Maffiuletti NA. Validity and reproducibility of the Physical Activity Scale for the Elderly (PASE) questionnaire for the measurement of the physical activity level in patients after total knee arthroplasty. BMC Musculoskelet Disord. 2014;15:46.

27. Schotanus MGM, Bemelmans YFL, Grimm B, Heyligers IC, Kort NP. Physical activity after outpatient surgery and enhanced recovery for total knee arthroplasty. Knee Surg Sports Traumatol Arthrosc. 2017;25(11):3366-3371.

28. Brandes M, Ringling M, Winter C, Hillmann A, Rosenbaum D. Changes in physical activity and health-related quality of life during the first year after total knee arthroplasty. Arthritis Care Res. 2011;63(3):328-334.

29. Lutzner C, Beyer F, Kirschner S, Lutzner J. How much improvement in patient activity can be expected after TKA? Orthopedics. 2016;39(3 Suppl):S18-S23.

30. Walker DJ, Heslop PS, Chandler C, Pinder IM. Measured ambulation and self-reported health status following total joint replacement for the osteoarthritic knee. Rheumatology (Oxford). 2002;41(7):755-758.

31. Tsonga T, Kapetanakis S, Papadopoulos C, et al. Evaluation of improvement in quality of life and physical activity after total knee arthroplasty in greek elderly women. Open Orthop J. 2011;5:343-347.

32. VanderZee KI, Sanderman R, Heyink J. A comparison of two multidimensional measures of health status: the Nottingham Health Profile and the RAND 36-Item Health Survey 1.0. Qual Life Res. 1996;5(1):165-174.

33. Moffet H, Collet JP, Shapiro SH, Paradis G, Marquis F, Roy L. Effectiveness of intensive rehabilitation on functional ability and quality of life after first total knee arthroplasty: a single-blind randomized controlled trial. Arch Phys Med Rehabil. 2004;85(4):546-556.

34. Monticone M, Ferrante S, Rocca B, et al. Home-based functional exercises aimed at managing kinesiophobia contribute to improving disability and quality of life of patients undergoing total knee arthroplasty: a randomized controlled trial. Arch Phys Med Rehabil. 2013;94(2):231-239.
35. Piva SR, Almeida GJ, Gil AB, DiGioia AM, Helsel DL, Sowa GA. A comprehensive behavioral and exercise intervention improves physical function and activity participation after total knee replacement - a pilot randomized study. Arthritis Care Res. 2017;69(12):1855-1862.

36. Godin G, Shephard RJ. A simple method to assess exercise behavior in the community. Can J Appl Sport Sci. 1985;10(3):141-146.

37. Almeida GJ, Terhorst L, Irrgang JJ, Fitzgerald GK, Jakicic JM, Piva SR. Responsiveness of physical activity measures following exercise programs after total knee arthroplasty. J Exerc Sports Orthop. 2017;4(3):1-8.

38. Ng M, Fleming $\mathrm{T}$, Robinson $\mathrm{M}$, et al. Global, regional and national prevalence of overweight and obesity in children and adults 1980-2013: a systematic analysis. Lancet. 2014;384(9945):766-781.

39. Lubbeke A, Zimmermann-Sloutskis D, Stern R, et al. Physical activity before and after primary total hip arthroplasty: a registry-based study. Arthritis Care Res. 2014;66(2):277-284.

40. Hammett T, Simonian A, Austin M, et al. Changes in physical activity after total hip or knee arthroplasty: a systematic review and metaanalysis of 6 and 12 month outcomes. Arthritis Care Res. 2017 Epub Sep 12 .

41. Swanson EA, Schmalzried TP, Dorey FJ. Activity recommendations after total hip and knee arthroplasty: a survey of the American Association for Hip and Knee Surgeons. J Arthroplasty. 2009;24(6 Suppl): 120-126.

42. Chase JA. Interventions to increase physical activity among older adults: a meta-analysis. Gerontologist. 2015;55(4):706-718.

43. Fanning J, Mullen SP, McAuley E. Increasing physical activity with mobile devices: a meta-analysis. J Med Internet Res. 2012;14(6):e161.

44. Bond DS, Thomas JG, Raynor HA, et al. B-MOBILE - a smartphone-based intervention to reduce sedentary time in overweight/ obese individuals: a within-subjects experimental trial. PLoS One. 2014;9(6):e100821.

45. Taylor WC, Hepworth JT, Lees E, et al. Readiness to change physical activity and dietary practices and willingness to consult healthcare providers. Health Res Policy Systems. 2004;2:2.

46. Jansink R, Braspenning J, Keizer E, van der Weijden T, Elwyn G, Grol R. Misperception of patients with type 2 diabetes about diet and physical activity, and its effects on readiness to change. $J$ Diabetes. 2012;4(4):417-423.

47. Sanchez A, Grandes G, Ortega Sanchez-Pinilla R, Torcal J, Montoya I. Predictors of long-term change of a physical activity promotion programme in primary care. BMC Public Health. 2014;14:108.
Open Access Journal of Sports Medicine

\section{Publish your work in this journal}

The Open Access Journal of Sports Medicine is an international, peer-reviewed, open access journal publishing original research, reports, reviews and commentaries on all areas of sports medicine. The journal is included on PubMed. The manuscript management system is completely online and includes a very quick and fair
Dovepress

peer-review system. Visit http://www.dovepress.com/testimonials.php to read real quotes from published authors. 